\title{
Nueva especie de Pseudoleucania Staudinger endémica para la Isla Robinson Crusoe (Chile) (Lepidoptera: Noctuidae: Noctuinae)
}

\section{A new species of Pseudoleucania Staudinger endemic to Robinson Crusoe Island (Chile) (Lepidoptera: Noctuidae: Noctuinae)}

\author{
luis E. Carrera-Suárez ${ }^{1 *}$, Andrés O. Angulo ${ }^{1}$ \& Tania S. Olivares ${ }^{2}$ \\ ${ }^{1}$ Departamento de Zoología, Facultad de Ciencias Naturales y Oceanográficas, Universidad de Concepción, casilla 160-C \\ Concepción, Chile. \\ ${ }^{2}$ Casilla 4040 correo 3. Concepción. Chile. \\ *E-mail: lucarrera@udec.cl
}

\begin{abstract}
RESUMEN
Se describe e ilustra el macho de Pseudoleucania masatierrae Carrera-Suárez \& Olivares n. sp. proveniente de la Isla Robinson Crusoe (= Más a Tierra), Archipiélago Juan Fernández, V Región, Chile. Se discute su distribución y la relación con la especie más próxima Pseudoleucania onerosa (Köhler).
\end{abstract}

Palabras clave: Taxonomía, Pseudoleucania onerosa, Más a Tierra, Archipiélago Juan Fernández.

\section{ABSTRACT}

The male of Pseudoleucania masatierrae Carrera-Suárez \& Olivares n. sp. from Robinson Crusoe Island (= Más a Tierra), Archipiélago Juan Fernández, V Región, Chile is described and illustrated. Its distribution and relationship with the closely related species Pseudoleucania onerosa (Köhler) are discused.

Keywords: Taxonomía, Pseudoleucania onerosa, Más a Tierra, Archipiélago Juan Fernández.

\section{INTRODUCCIÓN}

El género de lepidópteros nocturnos Pseudoleucania Staudinger, 1899 está compuesto por 59 especies; la mayoría de ellas tienen importancia agrícola (Angulo \& Olivares, 1992). Desde 1899 el género ha sido estudiado y varios géneros han sido creados para resolver su taxonomía, cabe mencionar Janaesia Angulo, Missio Angulo y Scania Olivares (Angulo, 1998).

La distribución de Pseudoleucania se encuentra en el dominio Andino-Patagónico de la Región Neotropical (Angulo \& Olivares, 1992). Hasta el momento, en el Archipiélago Juan Fernández no se han registrado nóctuidos pertenecientes a este taxón. En este trabajo se describe una nueva especie de Pseudoleucania de la Isla Robinson Crusoe (Archipiélago Juan Fernández, V Región, Chile).

\section{MATERIALES Y MÉTODOS}

El material revisado de la nueva especie proviene del Archipiélago Juan Fernández, Isla Robinson Crusoe (333' $42^{\prime \prime} \mathrm{S}$ y $\left.78^{\circ} 49^{\prime} 23^{\prime \prime} \mathrm{O}\right)$. La serie típica esta depositada en las Colecciones Científicas del Museo de Zoología (UCCC), Universidad de Concepción. Chile.

Se realizaron comparaciones con Pseudoleucania onerosa (Koehler): 1 Fototipo macho, Prov. Neuquén, Pucará, 1200 m, Paranicla onerosa nov., Det. Koehler, Leg B. Petrovski (ZSM; Zoologische Staatssammlung München, Alemania); y 1 macho, Magallanes, 13-marzo, 1991, Trampas coll. (UCCC).

La nomenclatura de la maculación alar y la estructura de la genitalia del macho usada en las descripciones sigue a 
Angulo \& Weigert (1977). Las fotografías digitales fueron hechas con una cámara digital Olympus C-5050.

\section{RESULTADOS}

Pseudoleucania masatierrae Carrera-Suárez \& Olivares n. sp. (Figs. 1, 2 y 3)

DESCRIPCIÓN EXTERNA DEL MACHO (Fig. 1): Cabeza y tórax caféclaro o crema; ojos glabros (desnudos); antenas fasciculadas; palpos con escamas piliformes castaño-claro y negro, del mismo largo que la cabeza. Alas anteriores castaño-claro; mancha reniforme de color castaño-oscuro, prácticamente tres veces la orbicular, en tamaño; mancha orbicular de color castaño-oscuro; mancha apical apenas presente, de color castaño-oscuro; venas concoloras; flecos en el borde del ala. Alas posteriores de color castaño-claro; venas concoloras; presenta flecos en el borde del ala; presencia de lúnula de color castaño-oscuro. Patagia y tegula concoloras al resto del cuerpo, con abundantes escamas piliformes; abdomen con escamas piliformes castaño-oscuro.

Descripción de la genitalia del MACho (Figs. 2 y 3 ): Uncus alargado con cuatro espinas en su ápice, las dos del centro más pequeñas que las externas; saccus puntiagudo hacia el ápice; valva espatulada, ancho del cucullus, la mitad el largo de la valva; una única hilera de espinas en la corona; ampulla curvada hacia la parte exterior de la valva, largo de la ampulla ca. de un tercio de la longitud de la valva; en la base de ésta, por la cara interna, presenta un pliegue con un largo ca. de un octavo de la ampulla; yuxta bilobular; vésica del aedeagus con tres espinas simples.

HEMBRA: desconocida.

ENVERGAdURA ALAR: machos: $\mathrm{X}=33 \mathrm{~mm}(\mathrm{n}=3)$.

Distribución: Chile: V Región. Isla Robinson Crusoe (=Más a Tierra).

Material examinado: (3 machos): 1 macho Holotipo, Archipiélago Juan Fernández. Pangal. 05-abril-1967. Cekalovic: coll; 1 macho Paratipo, Archipiélago Juan Fernández. Pangal. 05-abril-1967. Cekalovic: coll; 1 macho Paratipo, Archipiélago Juan Fernández, Isla Robinson Crusoe. Cerro Yunque. 17-diciembre-2008. E. Hernández \& P. Moreno: coll. Todos los ejemplares se encuentran depositados en las colecciones científicas de la Universidad de Concepción (UCCC).

Etimología: El epítope específico hace referencia al lugar de proveniencia del holotipo: Isla Robinson Crusoe (= Más a Tierra), Archipiélago Juan Fernández, V Región, Chile.

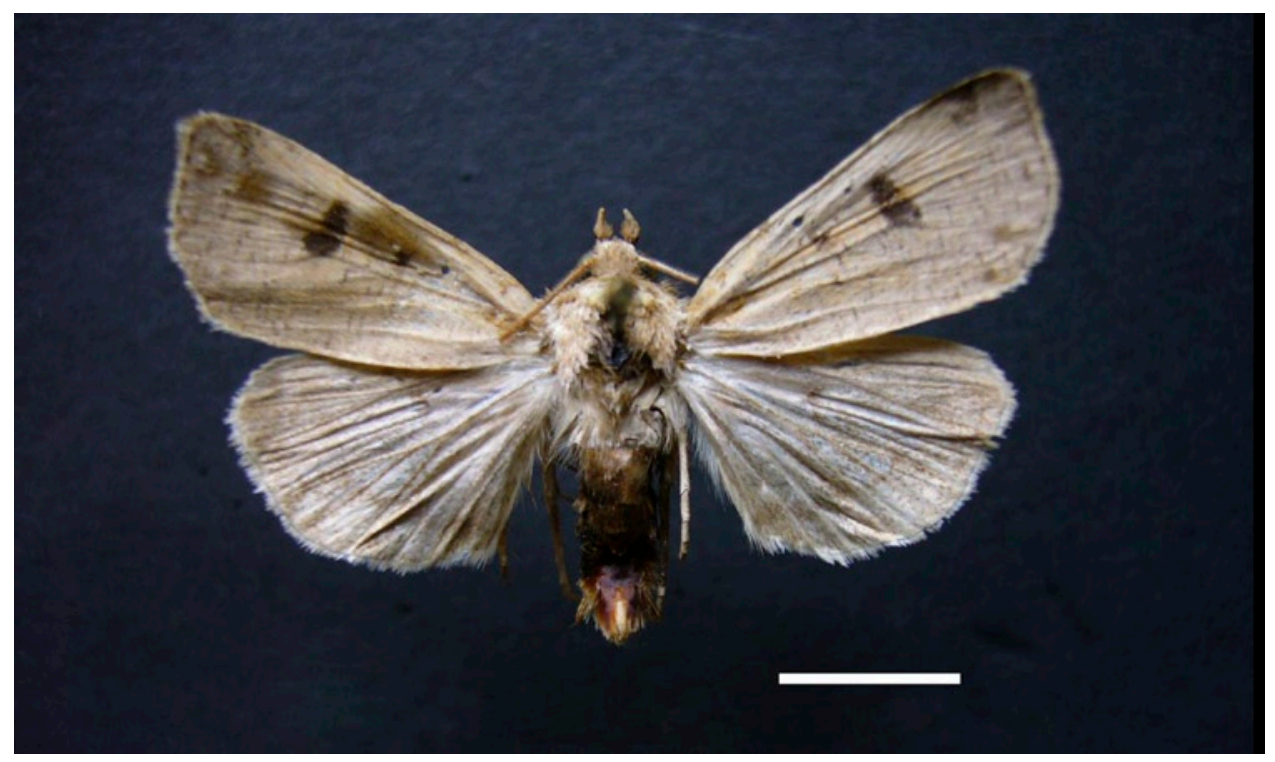

Figura 1: Holotipo de Pseudoleucania masatierrae Carrera-Suárez \& Olivares n. sp. (escala: $1 \mathrm{~cm}$ ). Figure 1: Holotype of Pseudoleucania masatierrae Carrera-Suárez \& Olivares n. sp. (scale: $1 \mathrm{~cm}$ ). 


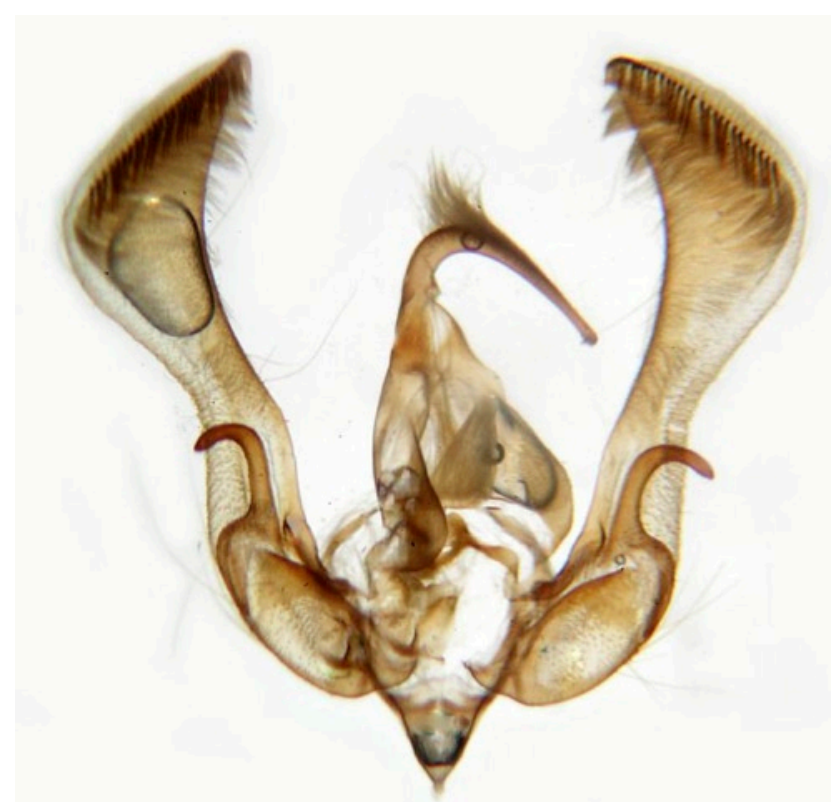

FIgURA 2: Genitalia del macho, valvas, de Pseudoleucania masatierrae Carrera-Suárez \& Olivares n. sp.

Figure 2: Male genitalia, valves, of Pseudoleucania masatierrae Carrera-Suárez \& Olivares n. sp.

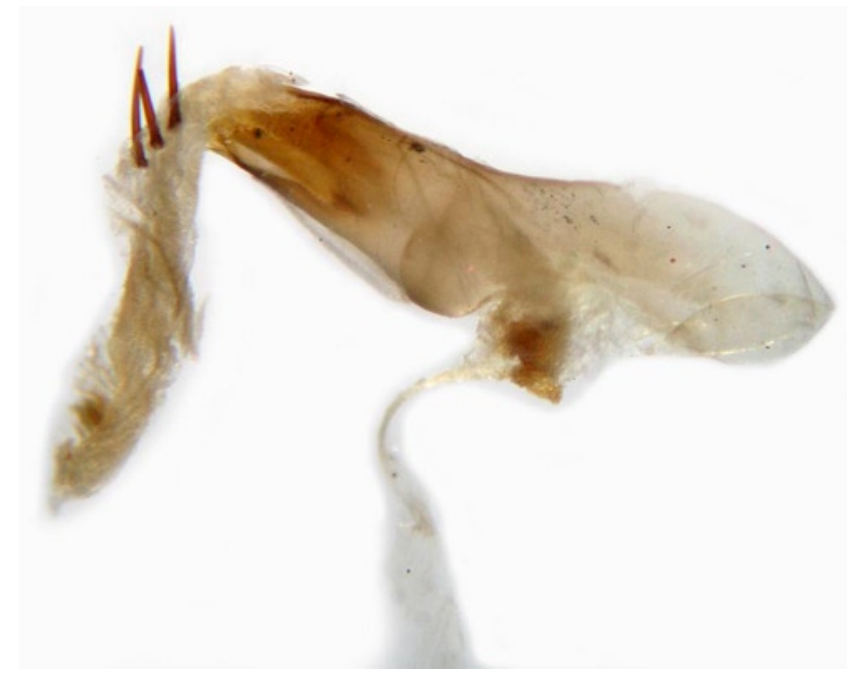

FIgUra 3: Genitalia del macho, aedeagus, de Pseudoleucania masatierrae Carrera-Suárez \& Olivares n. sp.

FIGURE 3: Male genitalia, aedeagus, of Pseudoleucania masatierrae Carrera-Suárez \& Olivares n. sp.

Comentarios taxonómicos: Pseudoleucania masatierrae Carrera-Suárez \& Olivares n. sp. se asemeja en su genitalia a Pseudoleucania onerosa (Köhler) (Figs. 4 y 5), pero en $P$. masatierrae n. sp. las valvas son más sinuosas; el saccus es puntiagudo en su ápice, mientras que en $P$. onerosa el saccus es redondeado. Pese a las variaciones intraespecíficas en la maculación alar de ambas especies, éstas se distinguen en el segundo par de alas: en $P$. masatierrae n. sp. son de color castaño-oscuro, mientras que en $P$. onerosa son blanquizcas en el caso de los machos. Es necesario mencionar que las descripciones se hicieron en base al holotipo; los paratipos presentan algunas variaciones: uno de los paratipos en la maculación alar no presenta la mancha reniforme ni la orbicular. En la genitalia la variación ocurre en el aedeagus, un paratipo presenta dos espinas simples y el otro es inerme; esta variación en las espinas es característica del género Pseudoleucania.

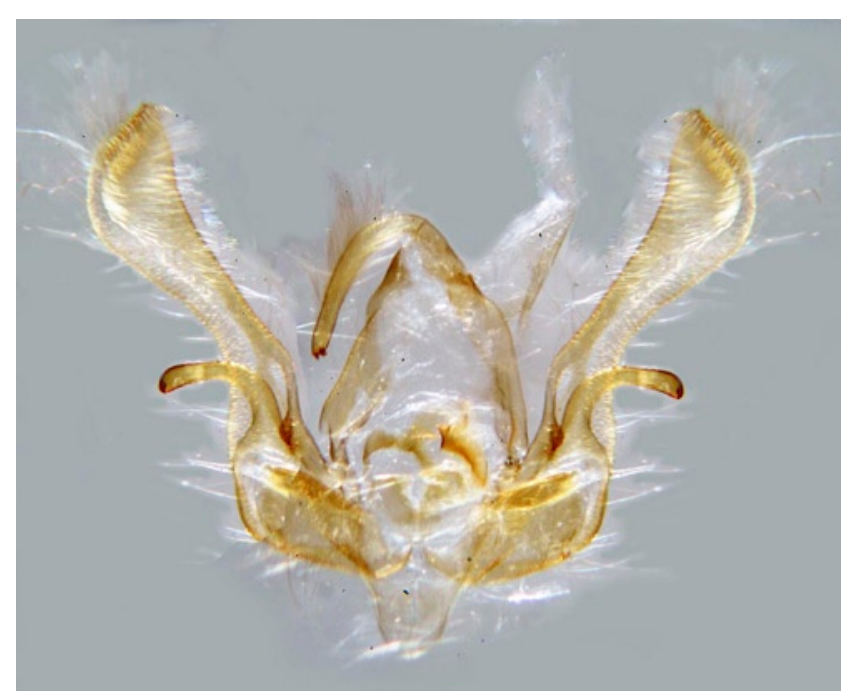

FIgURA 4: Genitalia del macho, valvas, de Pseudoleucania onerosa (Koehler, 1959).

FIgURE 4: Male genitalia, valves, of Pseudoleucania onerosa (Koehler, 1959).

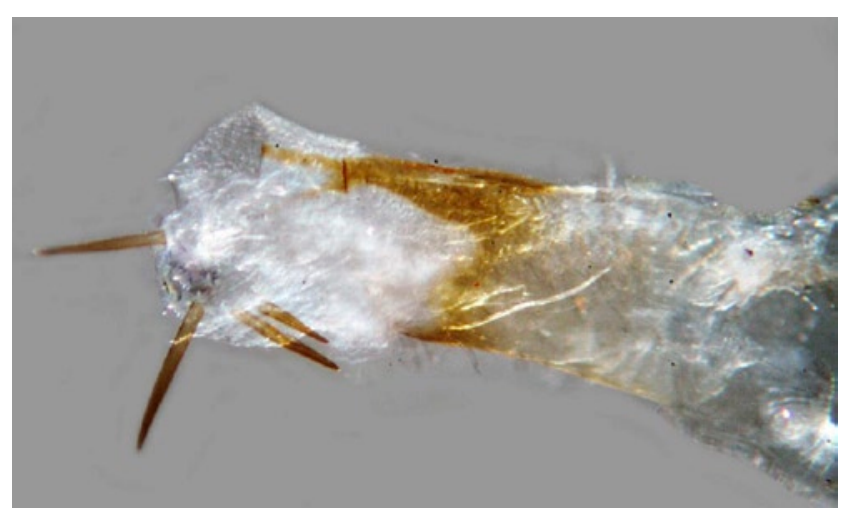

FIgura 5: Genitalia del macho, aedeagus, de Pseudoleucania onerosa (Koehler, 1959).

Figure 5: Male genitalia, aedeagus, of Pseudoleucania onerosa (Koehler, 1959). 
Gayana 74(1), 2010

\section{DISCUSIÓN}

Angulo (1998) reconoce 59 especies en el género Pseudoleucania; sin embargo, algunas de ellas han sido reubicadas en los géneros Scania Olivares, 1994 y Janaesia Angulo, 1998; quedando un número de 45 especies al que se debe agregar la nueva especie aquí descrita. Cabe destacar que Pseudoleucania masatierrae Carrera-Suárez \& Olivares n. sp. es una especie endémica de la Isla Robinson Crusoe. Aurivillius et al. (1922) describieron a Hoplotarsia magna (Noctuidae: Cuculliinae), la cual también sólo se encuentra en dicha isla. Por lo tanto, $P$. masatierrae n. sp. sería la segunda especie de lepidópteros nocturnos endémica para Robinson Crusoe. Al igual que lo que ocurre con muchas especies del género, respecto a $P$. masatierrae n. sp. se desconocen aspectos básicos de su biología, incluyendo los estados inmaduros.

\section{AGRADECIMIENTOS}

Agradecemos al Proyecto de Investigación de la Dirección de Investigación $\mathrm{N}^{\circ}$ 207.113.074-1.0 de la Universidad de Concepción y Al Dr. Gottfried Behounek de Die Zoologische Staatssammlung München (Alemania) por el envío de fototipos. Finalmente agradecemos al Dr. Fernando Navarro y a un revisor anónimo por sus comentarios.

\section{BIBLIOGRAFÍA}

Angulo, A.O. 1998. Revisión filogenia y clasificación de los géneros de Noctuinae Australes (Andino-Patagónicos) sudamericanos (Lepidoptera: Glossata: Noctuidae: Noctuinae). Gayana Zoológica 62 (1): 1-35.

Angulo, A.O. \& G. T.H. Weigert. 1977. Pseudaletia punctulata (Blanchard) y Pseudaletia impuncta (Guenée): nóctuidos hadeninos similares en Chile (Lepidoptera: Noctuidae). AgroSur 5(1): 12-17.

Angulo, A.O. \& T.S. Olivares. 1992. Una introducción al estudio del género Pseudoleucania Staudinger, 1899, en la subregión Andino-Patagónica (Lepidoptera: Noctuidae: Noctuinae). Investigación Agrícola (Chile) 12(1-2): 714.

Aurivillius, CHR., L.B. Prout \& E. Meyrick. 1922. Lepidopteren von Juan Fernandez und der Oster Insel. 256-260 pp. In: Skottsberg, C. The Natural History of Juan Fernández and Easter Island. 3:255-270, 2 plates. 